\title{
Cryogenic Fluid Management Technology Development for Nuclear Thermal Propulsion Abstract
}

The purpose of this paper is to investigate, facilitate a discussion and determine a path forward for technology development of cryogenic fluid management technology that is necessary for long duration deep space missions utilizing nuclear thermal propulsion systems. There are a number of challenges in managing cryogenic liquids that must be addressed before long durations missions into deep space, such as a trip to Mars can be successful. The leakage rate of hydrogen from pressure vessels, seals, lines and valves is a critical factor that must be controlled and minimized. For long duration missions, hydrogen leakage amounts to large increases in hydrogen and therefore vehicle mass. The size of a deep space vehicle, such as a mars transfer vehicle, must be kept small to control cost and the logistics of a multi launch, assembled in orbit vehicle. The boil off control of the cryogenic fluid is an additional obstacle to long duration missions. The boil off caused by heat absorption results in the growth of the propellant needs of the vehicle and therefore vehicle mass. This is a significant problem for a vehicle using nuclear (fission) propulsion systems. Radiation from the engines deposits

large quantities of heat into the cryogenic fluid, greatly increasing boil off beyond that caused by environmental heat leakage. Addressing and resolving these challenges is critical to successful long duration space exploration. This paper discusses the state of the technology needed to address these challenges and discuss the path forward needed in technology development. 